\title{
New nonlinear multivariable model shows the relationship between central corneal thickness and HRTII topographic parameters in glaucoma patients
}

\author{
Dimitrios Kourkoutas ${ }^{1,2}$ \\ Gerasimos Georgopoulos' \\ Antonios Maragos' \\ Ioannis Apostolakis' \\ George Tsekouras ${ }^{4}$ \\ Irene S Karanasiou ${ }^{3}$ \\ Dimitrios Papaconstantinou' \\ Evaggelos Iliakis' \\ Michael Moschos' \\ 'Department of Ophthalmology, \\ Medical School, Athens University, \\ Athens, Greece; ${ }^{2}$ Department \\ of Ophthalmology, 417 Hellenic \\ Army Shared Fund Hospital, Athens, \\ Greece; ${ }^{3}$ Microwave and Fibre Optics \\ Laboratory, School of Electrical \\ and Computer Engineering, National \\ Technical University of Athens, Athens, \\ Greece; ${ }^{4}$ Department of Electrical \\ Engineering and Computer Science, \\ Hellenic Naval Academy, Piraeus, \\ Greece
}

Correspondence: Dimitrios Kourkoutas Department of Ophthalmology,

417 Hellenic Army Shared Fund Hospital,

10-12 M Petraki Street,

II52I, Athens, Greece

Tel +30210728800I Ext. 433

Email d_kourkoutas@hotmail.com
Purpose: In this paper a new nonlinear multivariable regression method is presented in order to investigate the relationship between the central corneal thickness (CCT) and the Heidelberg Retina Tomograph (HRTII) optic nerve head (ONH) topographic measurements, in patients with established glaucoma.

Methods: Forty nine eyes of 49 patients with glaucoma were included in this study. Inclusion criteria were patients with (a) HRT II ONH imaging of good quality (SD $<30 \mu \mathrm{m}$ ), (b) reliable Humphrey visual field tests (30-2 program), and (c) bilateral CCT measurements with ultrasonic contact pachymetry. Patients were classified as glaucomatous based on visual field and/or ONH damage. The relationship between CCT and topographic parameters was analyzed by using the new nonlinear multivariable regression model.

Results: In the entire group, CCT was $549.78 \pm 33.08 \mu \mathrm{m}$ (range: $484-636 \mu \mathrm{m}$ ); intraocular pressure (IOP) was $16.4 \pm 2.67 \mathrm{mmHg}$ (range: $11-23 \mathrm{mmHg}$ ); $\mathrm{MD}$ was $-3.80 \pm 4.97 \mathrm{~dB}$ (range: $4.04-[-20.4] \mathrm{dB}$ ); refraction was $-0.78 \pm 2.46 \mathrm{D}$ (range: $-6.0 \mathrm{D}$ to $+3.0 \mathrm{D}$ ). The new nonlinear multivariable regression model we used indicated that CCT was significantly related $\left(\mathrm{R}^{2}=0.227\right.$, $\mathrm{p}<0.01$ ) with rim volume nasally and type of diagnosis.

Conclusions: By using the new nonlinear multivariable regression model, in patients with established glaucoma, our data showed that there is a statistically significant correlation between CCT and HRTII ONH structural measurements, in glaucoma patients.

Keywords: central corneal thickness, glaucoma, optic nerve head, HRT

\section{Introduction}

Central corneal thickness (CCT) has been implicated as a risk factor for the development of primary open-angle glaucoma (POAG) and the development of glaucomatous visual field (VF) defects among ocular hypertensive patients ${ }^{1,2}$ and patients with preperimetric glaucomatous optic neuropathy. ${ }^{3} \mathrm{CCT}$ has also been associated with VF progression in patients with POAG. ${ }^{4}$

However, it has been reported that clinically detectable glaucomatous structural alteration of the $\mathrm{ONH}$ may precede the development of reproducible white on white $^{5-9}$ and blue on yellow ${ }^{8-10}$ VF defects by up to several years. Correspondingly, an investigation by Herndon and colleagues ${ }^{11}$ found that CCT was the most consistent predictor of the degree of glaucomatous optic nerve head $(\mathrm{ONH})$ structural damage. Most recently, Hewitt and colleagues ${ }^{12}$ reported that, in glaucomatous eyes, thinner CCT was related to increased severity of optic disc cupping. In this study, the corrected vertical cup-to-disc ratio (VCDR) was used as the structural marker of glaucoma severity and was calculated by using a modified $60 \mathrm{D}$ lens. ${ }^{13}$ The same results were 
found by Jonas and colleagues by evaluating $\mathrm{ONH}$ color stereophotographs. ${ }^{14}$ Although such quantitative $\mathrm{ONH}$ evaluations have been developed, most of them are complex and time consuming. The advent of computerized instruments such as the Heidelberg Retina Tomograph (HRT; Heidelberg Engineering, GmbH, Dossenheim, Germany) have introduced rapid, quantitative three dimensional analysis of the $\mathrm{ONH}$ and retinal nerve fiber layer (RNFL). The HRT provides rapid, objective and reproducible ${ }^{15-17}$ measurements of numerous ONH and RNFL stereometric parameters.

Additionally, due to the continuity of the cornea, sclera and optic disc lamina, CCT may represent a factor that reflects the biomechanics of the ONH even though we do not know the exact relationship between ONH susceptibility and CCT. Several researchers have turned to numerical modeling to understand the biomechanical environment within the ONH. ${ }^{18-20}$ The most interesting prediction they made was that the biomechanics of the corneoscleral shell affect cellular deformation in the $\mathrm{ONH}$ quite profoundly. Therefore, it seems reasonable that there should be a relationship between the CCT and biomechanical properties of the cornea and those of the sclera and $\mathrm{ONH}$. We may therefore consider the possibility that CCT may be extrapolated to topographic characteristics and parameters of the optic disc itself.

The purpose of this study was to investigate the association between the CCT and the quantitative ONH topographic parameters as measured by the HRTII in patients with established glaucoma using a new nonlinear multivariable regression model..$^{21-23}$

\section{Patients and methods}

The study population consisted of patients with documented open angle glaucoma - according to patients' charts - being followed at the outpatient clinics of the Glaucoma Unit at the University of Athens. Caucasian patients with open angle glaucoma were consecutively recruited between August and December 2005 without knowing the severity of VF defects and ONH damage. Glaucoma diagnoses included were POAG, pseudoexfoliative glaucoma (PXF), pigmentary glaucoma (PG), and normal tension glaucoma (NTG). Informed consent was obtained from all patients after the examination procedure was fully explained. The study protocol was designed according to the Declaration of Helsinki and approved by our Institutional Review Board.

Patients were included if they were aged 35-80 years, had best-corrected visual acuity (BCVA) better than 20/40, open anterior chamber angle, spherical refractive errors $<+6.00$ and $>-6.00 \mathrm{D}$ and cylinder $<3.00 \mathrm{D}$, previous experience of full threshold perimetry, reliable Humphrey field analyzer (HFA) VFs (fixation losses, false positives, and false negatives $<25 \%$ ) and good image quality with the HRT (SD $<30 \mu \mathrm{m}$ ). One eye from each patient was randomly selected to be included in the study.

The exclusion criteria included neurological disease, history of ocular trauma, history of stroke or diabetic retinopathy, corneal opacification of any etiology, use of contact lenses, previous corneal laser or surgery, less than six months post-cataract or post-glaucoma surgery, any history of disease or use of medication that may affect VF reliability, and a suspicion or actual defect in the VF of the eye being tested that is explained by the patient's ocular status or history, other than glaucoma.

All patients underwent complete ophthalmologic examination, CCT, automated VF test, and ONH tomography. The complete ophthalmologic examination included BCVA, slit-lamp biomicroscopy, Goldmann applanation tonometry (GAT), gonioscopy, and dilated indirect ophthalmoscopy for optic disc and RNFL evaluations.

VF tests were performed with the HFA (Model 740, Humphrey-Zeiss, Dublin, CA, USA) using the full threshold 30-2 program.

ONH tomography was performed using the HRT (software version 2.01). CCT was measured with an ultrasonic pachymeter (Echoscan US-1800, Nidek Co., Japan).

CCT was measured with an ultrasonic pachymeter. The pachymeter probe was placed on the centre of the cornea and the mean of five readings was automatically calculated for each eye.

GAT was performed on a slit lamp (Haag- Streit, Köniz, Switzerland) with a calibrated tonometer. Before each reading, the measuring drum was reset to approximately $10 \mathrm{mmHg}$, and the mean of three consecutive readings was recorded. It should be noted that the IOP used in this study was under treatment and was not corrected for CCT.

All measurements were taken during the same visit in the following order: VF test, ONH tomography, CCT, and GAT.

For the purpose of this study, the diagnosis of glaucoma was confirmed by a glaucoma specialist using the following information: ${ }^{24}$

1. Existence of VF defects

a. Abnormal glaucoma hemifield test, confirmed in two consecutive tests

b. Three abnormal points confirmed on two consecutive tests, with $\mathrm{p}<5 \%$ of being normal, one of which should have $\mathrm{p}<1 \%$, all being contiguous with the blind spot 
c. Corrected pattern standard deviation $<5 \%$ if the VF is otherwise normal, confirmed on two consecutive tests and/or

2. Existence of glaucomatous optic disc abnormalities.

\section{Statistical methods}

A new nonlinear multivariable regression model was developed to determine the relationship between CCT and HRTII ONH topographic measurements. ${ }^{21-23}$ All the HRTII global parameters, 36 sectoral parameters, Moorfields regression analysis (MRA) as well as independent parameters (age, VF mean defect [MD], refraction, IOP, and diagnosis) were used with this model.

The specific logistic model used in the present study has not been applied before in the ophthalmology literature and we describe it briefly. It is mentioned that the physical systems are rarely linear and this approximation usually leads to no representative models. The proposed model has been already applied for the quantitative solution of different nonlinear physical and engineering problems, such as data mining, ${ }^{21}$ statistical indices, load and energy forecasting, ${ }^{22,23}$ estimation of the settlements during the construction of a tunnel, etc. Its basic advantages (against previous models) are the capability to use of more than one independent variables and the identification of the nonlinear relationships between them.

In summary, this method performs an extensive search in order to select the most appropriate functions and weighting factors to be used in the model, following the basic steps as described by Tsekouras and colleagues (Figure 1; Appendix): $:^{22,23}$

a) Proper transformation of the model variables

b) Use of correlation analysis

c) Model optimization regarding the selection of input variables and the application of the validation criteria. The validation criteria are the F-test, the coefficient determination $\mathrm{R}^{2}$ for the regression model, and $\mathrm{t}$-tests.

\section{Results}

During the study period, 49 eyes of 49 patients were eligible and were included in the study. 29 patients were females and 20 were males. The mean age of study patients was $61.9 \pm 12.01$ years (range: $35-79$ years). Five patients were diagnosed with NTG, 5 patients with PG, 10 patients with PXF, and 29 patients with POAG. The mean CCT for the whole sample was $549.8 \pm 33.08 \mu \mathrm{m}$ (range: 484-636 $\mu \mathrm{m}$ ). Subjects of our study had a mean VF MD of $-3.80 \pm 4.97 \mathrm{~dB}$ (range: $-20.40-4.04 \mathrm{~dB}$ ). The mean

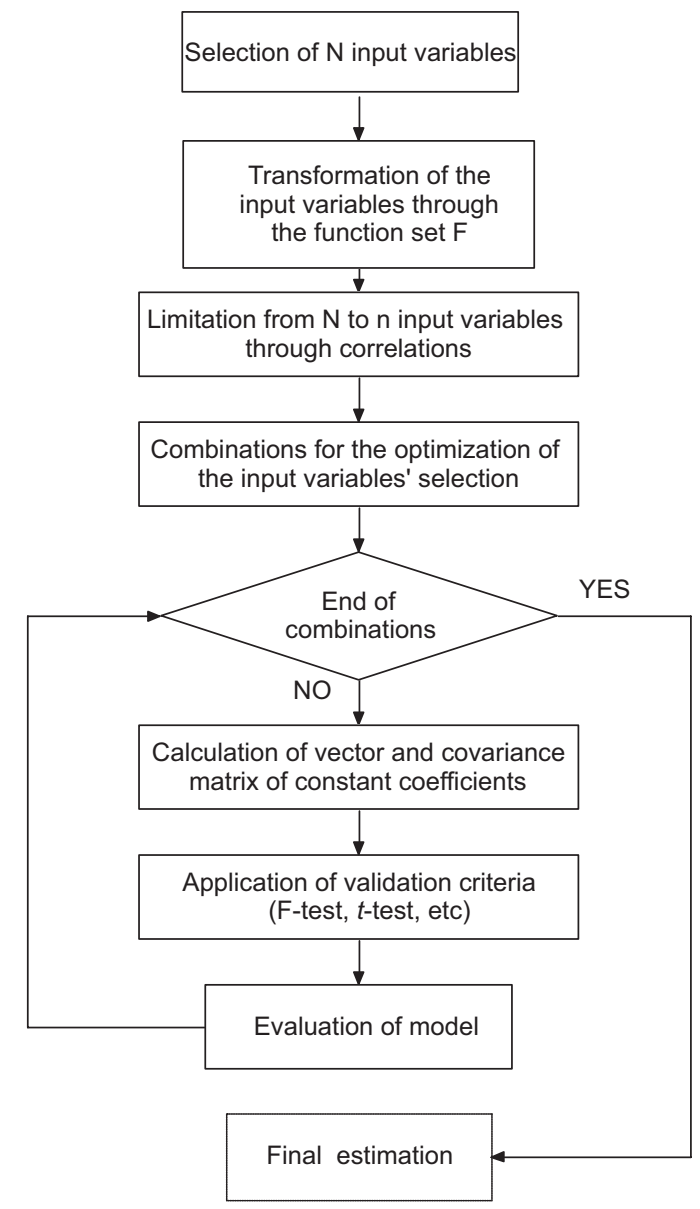

Figure I Developed nonlinear multivariable regression model.

IOP was $16.4 \pm 2.67 \mathrm{mmHg}$ (range: $11-23 \mathrm{mmHg}$ ). The mean spherical equivalent refraction was $-0.78 \pm 2.46 \mathrm{D}$ (range: -6.0-3.0 D). Descriptive statistics per type of glaucoma (mean $\pm \mathrm{SD}$ ) are presented in Table 1 and Figure 3.

By using the new nonlinear multivariable regression model, the following equation for CCT was statistically significant $(\mathrm{p}<0.01)$ :

$$
\begin{aligned}
\mathrm{CCT}= & 548.69+131.78 \cdot[\mathrm{rim} \text { volume nas }]^{0.5} \\
& -28.676 \cdot[\text { diagnosis }]^{0.5}
\end{aligned}
$$

The respective validation criteria have the following values:

$$
\begin{aligned}
F & =6.762>F_{p_{1}=0.01 \cdot v_{2}=48 \cdot v_{1}=2}=5.0767 \\
t_{b_{1}} & =17.8736>t_{p_{2}=0.0001 \cdot v_{1}=46}=4.2601 \\
t_{b_{2}} & =2.6022>t_{p_{2}=0.05 \cdot v_{1}=46}=2.0129 \\
t_{b_{3}} & =-1.8516 \Rightarrow t_{b_{3}}=1.8516>t_{p_{2}=0.05 \cdot v_{1}=46} \\
& =1.6787 \\
R^{2} & =0.227
\end{aligned}
$$


Table I Descriptive statistics per type of glaucoma (mean \pm SD)

\begin{tabular}{|c|c|c|c|c|c|}
\hline Parameter & Total & POAG & PXF & NTG & PG \\
\hline Eyes (n) & 49 & 29 & 10 & 5 & 5 \\
\hline CCT $(\mu \mathrm{m})$ & $549.8( \pm 33.08)$ & $551.06( \pm 31.82)$ & $565.60( \pm 4 \mid .42)$ & $530.40( \pm 19.47)$ & $530.40( \pm 15.10)$ \\
\hline IOP (mmHg) & $16.4( \pm 2.66)$ & $16.82( \pm 2.91)$ & $14.90( \pm 1.52)$ & $16.20( \pm 2.48)$ & $17.20( \pm 2.58)$ \\
\hline Age (years) & $61.97( \pm 12.01)$ & $62.3( \pm 11.48)$ & $70.4( \pm 6.25)$ & $57.40( \pm 10.5)$ & $47.8( \pm 12.47)$ \\
\hline$M D(d B)$ & $-3.80( \pm 4.96)$ & $-3.39( \pm 4.83)$ & $-3.07( \pm 2.19)$ & $-10.38( \pm 7.36)$ & $-1.04( \pm 1.02)$ \\
\hline Disc area $\left(\mathrm{mm}^{2}\right)$ & $2.131( \pm 0.385)$ & $2.16 \mathrm{I}( \pm 0.365)$ & $1.98 \mid( \pm 0.277)$ & $2.465( \pm 0.589)$ & $1.922( \pm 0.253)$ \\
\hline Cup area $\left(\mathrm{mm}^{2}\right)$ & $0.788( \pm 0.493)$ & $0.763( \pm 0.467)$ & $0.654( \pm 0.4 I 0)$ & $1.410( \pm 0.503)$ & $0.577( \pm 0.4 \mid 3)$ \\
\hline Cup/Disc area ratio & $0.353( \pm 0.189)$ & $0.339( \pm 0.184)$ & $0.318( \pm 0.186)$ & $0.566( \pm 0.121)$ & $0.291( \pm 0.185)$ \\
\hline Rim/Disc area ratio & $0.646( \pm 0.189)$ & $0.660( \pm 0.184)$ & $0.681( \pm 0.186)$ & $0.433( \pm 0.121)$ & $0.709( \pm 0.185)$ \\
\hline Cup volume $\left(\mathrm{mm}^{3}\right)$ & $0.249( \pm 0.273)$ & $0.248( \pm 0.291)$ & $0.166( \pm 0.166)$ & $0.502( \pm 0.323)$ & $0.165( \pm 0.170)$ \\
\hline Rim volume $\left(\mathrm{mm}^{3}\right)$ & $0.315( \pm 0.143)$ & $0.327( \pm 0.136)$ & $0.325( \pm 0.153)$ & $0.183( \pm 0.085)$ & $0.360( \pm 0.172)$ \\
\hline CSM & $-0.124( \pm 0.080)$ & $-0.131( \pm 0.076)$ & $-0.143( \pm 0.074)$ & $-0.018( \pm 0.055)$ & $-0.153( \pm 0.067)$ \\
\hline Linear C/D ratio & $0.570( \pm 0.167)$ & $0.56 \mathrm{I}( \pm 0.158)$ & $0.535( \pm 0.186)$ & $0.749( \pm 0.080)$ & $0.516( \pm 0.173)$ \\
\hline FSM & $0.252( \pm 1.978)$ & $0.519( \pm 1.838)$ & $0.873( \pm \mathrm{I} .888)$ & $-2.509( \pm 1.139)$ & $0.226( \pm 1.733)$ \\
\hline MRA* & $1.69( \pm 0.82)$ & $1.48( \pm 0.68)$ & $1.70( \pm 0.82)$ & $2.8( \pm 0.44)$ & $1.80( \pm 1.09)$ \\
\hline
\end{tabular}

Notes: *The values of Moorfields regression analysis can be within normal limits, borderline, outside normal limits, which are represented by the arithmetic values I to 3 , respectively.

Abbreviations: CCT, central corneal thickness; CSM, cup shape measure; IOP, intraocular pressure; FSM, Frederick S Mikelberg discriminant function; MD, mean defect; MRA, Moorfields regression analysis; NTG, normal tension glaucoma; PG, pigmentary glaucoma; POAG, primary open-angle glaucoma; PXF, pseudoexfoliative glaucoma.

The results of simple linear monovariable regression model between each input variable and CCT using the $R^{2}$ criterion are presented in Table 2.

\section{Discussion}

The present study showed a statistically significant association between CCT and certain HRTII quantitative $\mathrm{ONH}$ topographic parameters, in patients with established glaucoma.

From the application of the nonlinear multivariable regression model the respective equation (1) had a quite satisfactory coefficient determination $\left(R^{2}=0.227\right)$ using the variables rim volume nasally and type of diagnosis, with $p<0.01$.

The CCT was positively correlated to rim volume nasally and negatively correlated to the type of diagnosis. It is noted that the dependence of CCT on the nasal rim volume is quite strong, because the respective $t$-test is also satisfied with $p<0.0001$.

This model therefore indicates that a larger CCT is strongly related with a larger rim volume in the nasal sector of the optic disc. It is well documented in the literature that glaucomatous neuroretinal rim loss takes place in a sequence of sectors that correlates with the progression of visual field defects and the morphology of the lamina cribrosa. ${ }^{25}$ Generally, it begins in the inferotemporal disc region and then progresses to the superotemporal, and the temporal sectors. Usually, rim remnants are present in the nasal sector until the advanced glaucoma stages. While the importance of the superotemporal and inferotemporal disc sectors for glaucoma diagnosis has already been shown in previous studies, ${ }^{26-28}$ the importance of the nasal optic disc sector has not been clearly demonstrated yet.

Our developed model also includes the variable diagnosis. The values of diagnosis in the current study can be PXF, POAG, PG, NTG, which are represented by the arithmetic values 1 to 4 respectively. This variable is omitted in the cases of different models for each kind of glaucoma diagnosis. Our model therefore confirms the observation that mean CCT differs within glaucoma subgroups (Table 1; Figure 3). It is reported in the literature that the mean CCT in NTG is lower than in POAG. ${ }^{29,30}$

The results of simple linear monovariable regression model between each input variable and CCT are presented in Table 2. There was a statistically significant correlation $(\mathrm{p}<0.05)$ between certain HRTII ONH parameters and CCT in our group of eyes, with $\mathrm{R}^{2}$ ranging from 0.0804 to 0.1213 . In a recent study, CCT was inversely correlated to optic disc area in patients with POAG. ${ }^{31}$ The current study found no relationship between CCT and optic disc size. In the present study, CCT was positively correlated to rim area nasal, rim/ disc area ratio, rim volume nasal, and Frederick S Mikelberg 


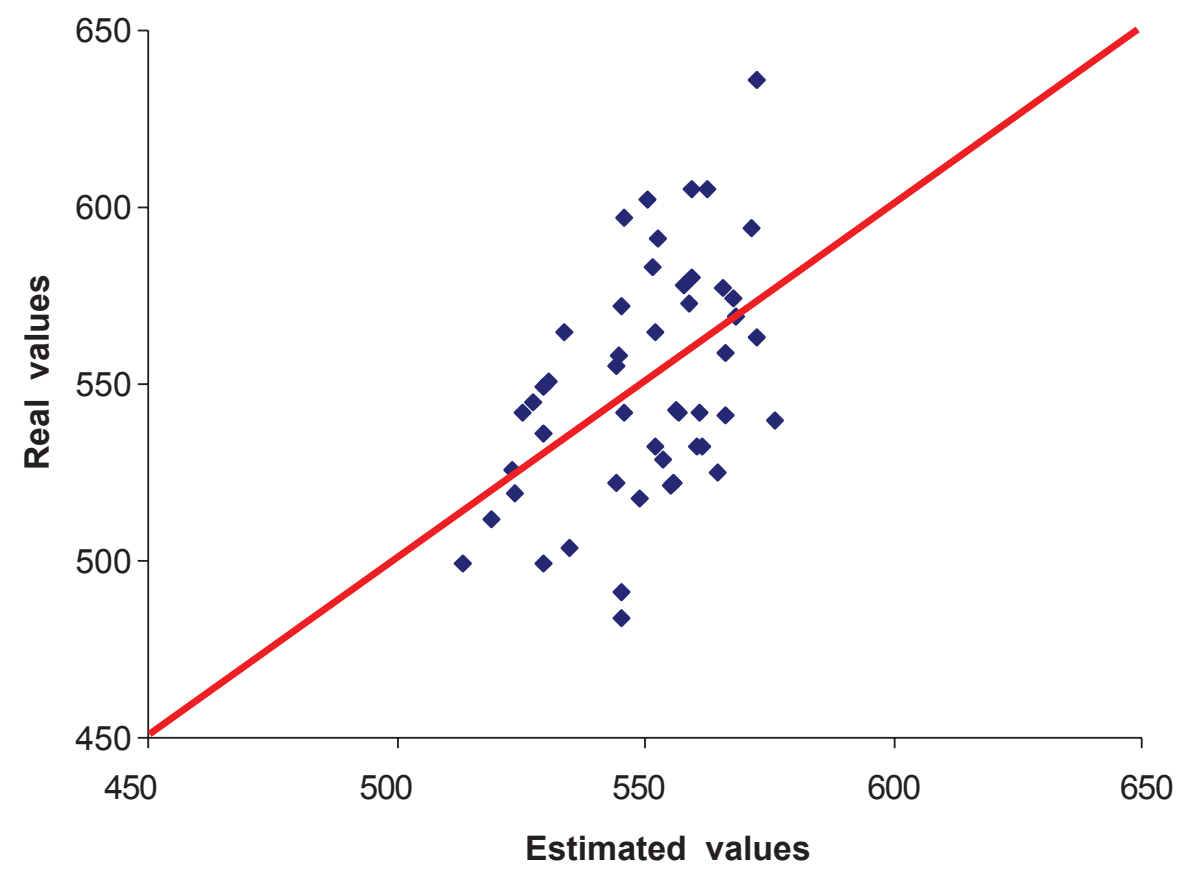

Figure 2 Actual and estimated values of the central corneal thickness using the nonlinear multivariable regression model for the data set of 49 eyes measured.

discriminant function. The CCT was also negatively correlated to cup area, cup/disc area ratio, cup volume, cup area temporal/inferior, cup area nasal, cup area nasal/inferior, cup volume temporal, cup volume temporal/inferior, cup volume nasal, cup volume nasal/inferior, type of diagnosis, and MRA. The MRA values can be within "normal limits", "borderline", "outside normal limits", which are represented by the arithmetic values 1 to 3 respectively.

Therefore, by using the HRTII ONH structural measurements, we confirm the results of Herndon and colleagues ${ }^{11}$ who examined consecutive patients with POAG at the first presentation to a glaucoma specialist and first found that CCT was a consistent predictor of the degree of glaucomatous damage. Hewitt and colleagues ${ }^{12}$ also reported that, in glaucomatous eyes, thinner CCT was related to increased corrected VCDR, by using a modified $60 \mathrm{D}$ lens. ${ }^{13}$ The same results were found by Jonas and colleagues, by evaluating ONH color stereophotographs. ${ }^{14}$ The limitation of these studies is that the assessment of ONH was necessarily subjective and thus potentially prone to greater error. The advent of HRT, with good reproducibility ${ }^{15-17}$ as well as high sensitivity and specificity ${ }^{32,33}$ in glaucoma diagnosis, provides us with objective ONH structural measurements for investigating quantitative associations. However, it should be noted that this technique is based on the contour line drawn by the operator and the capacity of the system to set a reference plane $50 \mu \mathrm{m}$ below the retinal surface height between $350^{\circ}$ and $356^{\circ}$. The reference plane is theoretically located within the papillomacular bundle, which is the least involved part in glaucomatous damage. ${ }^{34}$ Nevertheless, the position of this plane can change from one patient to another and may subsequently affect the analysis of $\mathrm{ONH}$ structural measurements.

In a parallel manner, the results of the present study suggest that patients who are followed in a glaucoma unit and suffer from open angle glaucoma have more advanced glaucomatous optic nerve damage if the cornea is relatively thin than if the cornea is relatively thick.

Interestingly, CCT was not correlated to the uncorrected applanation IOP at the time of study enrolment (Eq. 1; Table 2). It should be noted that although IOP was measured, it was not considered to be a major outcome factor because all the subjects were receiving treatment prior to study recruitment. This result is inconsistent with previous studies that showed the CCT was larger in subjects with ocular hypertension compared to normotensive subjects. ${ }^{35-42}$ It is therefore well documented in the literature that applanation IOP is influenced by CCT. Nonetheless, a study such as this, where patients have commenced medical and/or surgical treatment many years prior to enrolment without taking into account the CCT factor, may fail to disclose a relevant relationship between CCT and IOP. The management of each patient aims to reduce glaucoma progression through lowering IOP to individualized levels (target IOP). Therefore, the 
Table $2 R^{2}$ criterion between the experimental and the predicted values of CCT for simple linear regression model of one input variable

\begin{tabular}{|c|c|c|c|c|c|}
\hline Parameter & $R^{2}$ & F-test & t-test & F-test (satisfied) & t-test (satisfied) \\
\hline Age & 0.0032 & 0.1506 & -0.3887 & No & No \\
\hline Md & 0.0491 & 2.3063 & 1.5573 & No & No \\
\hline Refraction & 0.0008 & 0.0390 & 0.1976 & No & No \\
\hline IOP & 0.0093 & $0.437 \mid$ & 0.6643 & No & No \\
\hline Disc area & 0.0242 & 1.1385 & -1.0802 & No & No \\
\hline Cup area & 0.1033 & 4.8547 & -2.3268 & $\mathrm{P}<0 . \mathrm{I}$ & $\mathrm{P}<0.05$ \\
\hline Rim area & 0.0777 & 3.6515 & 1.9897 & $\mathrm{P}<0 . \mathrm{I}$ & $\mathrm{P}<0 . \mathrm{I}$ \\
\hline Rim area temporal & 0.0550 & 2.5867 & 1.6545 & No & $P \approx 0.1$ \\
\hline Rim area temp/sup & 0.0203 & 0.9534 & 0.9865 & No & No \\
\hline Rim area temp/inf & 0.0314 & 1.4780 & 1.2353 & No & No \\
\hline Rim area nasal & 0.1534 & 7.2109 & 2.9185 & $\mathrm{P}<0.01$ & $\mathrm{P}<0.0 \mathrm{I}$ \\
\hline Rim area nas/sup & 0.0181 & 0.8498 & 0.9303 & No & No \\
\hline Rim area nas/inf & 0.0341 & 1.6026 & $1.288 \mathrm{I}$ & No & No \\
\hline Cup/disc area ratio & 0.1099 & 5.1630 & -2.4083 & $\mathrm{p}<0.05$ & $\mathrm{p}<0.05$ \\
\hline $\mathrm{Rim} /$ disc area ratio & 0.1099 & 5.1630 & 2.4083 & $\mathrm{P}<0.05$ & $\mathrm{P}<0.05$ \\
\hline Cup volume & 0.1095 & 5.1485 & -2.4045 & $\mathrm{P}<0.05$ & $\mathrm{P}<0.05$ \\
\hline Rim volume & 0.0706 & 3.3172 & 1.8892 & $\mathrm{P}<0 . \mathrm{I}$ & $\mathrm{P}<0 . \mathrm{I}$ \\
\hline Rim volume temporal & 0.0683 & 3.2098 & $\mathrm{I} .856 \mathrm{I}$ & $\mathrm{p}<0 . \mathrm{I}$ & $\mathrm{P}<0.1$ \\
\hline Rim volume temp/sup & 0.0201 & 0.9444 & 0.9817 & No & No \\
\hline Rim volume temp/inf & 0.0178 & $0.837 \mid$ & 0.9232 & No & No \\
\hline Rim volume nasal & 0.1551 & 7.2884 & 2.9370 & $\mathrm{P}<0.01$ & $\mathrm{P}<0.01$ \\
\hline Rim volume nas/sup & 0.0115 & 0.5424 & 0.7408 & No & No \\
\hline Rim volume nas/inf & 0.0341 & 1.6031 & 1.2883 & No & No \\
\hline Min cup depth & 0.0388 & 1.8238 & -1.3775 & No & No \\
\hline Max cup depth & 0.0061 & 0.2872 & -0.5376 & No & No \\
\hline Height variation contour & 0.0180 & 0.8467 & 0.9286 & No & No \\
\hline CSM & 0.0648 & 3.0454 & -1.8046 & $\mathrm{P}<0 . \mathrm{I}$ & $\mathrm{P}<0 . \mathrm{I}$ \\
\hline Mean RNFL thickness & 0.0518 & 2.4354 & 1.6026 & No & No \\
\hline RNFL thickness temporal & 0.0307 & 1.4439 & 1.2205 & No & No \\
\hline RNFL thickness temp/sup & 0.0239 & 1.1238 & 1.0730 & No & No \\
\hline RNFL thickness temp/inf & 0.0756 & 3.5555 & 1.9612 & $\mathrm{P}<0 . \mathrm{I}$ & $\mathrm{P}<0 . \mathrm{I}$ \\
\hline RNFL thickness nasal & 0.0495 & 2.3285 & 1.5652 & No & No \\
\hline RNFL thickness nas/sup & 0.0306 & 1.4384 & 1.2181 & No & No \\
\hline RNFL thickness nas/inf & 0.0259 & 1.2186 & 1.1185 & No & No \\
\hline RNFL cross sectional area & 0.0432 & 2.0323 & 1.4575 & No & No \\
\hline Linear $\mathrm{c} / \mathrm{d}$ ratio & 0.0783 & 3.6815 & -1.9986 & $\mathrm{P}<0 . \mathrm{I}$ & $\mathrm{P}<0.1$ \\
\hline Max. Contour elevation & 0.0227 & 1.0662 & -1.0445 & No & No \\
\hline Max. Contour depression & 0.0001 & 0.0070 & -0.0836 & No & No \\
\hline CLM temporal superior & 0.0178 & 0.8388 & 0.9241 & No & No \\
\hline CLM temporal inferior & 0.0702 & 3.2971 & $\mathrm{I} .8830$ & $\mathrm{P}<0 . \mathrm{I}$ & $\mathrm{P}<0 . \mathrm{I}$ \\
\hline Average variability SD & 0.0007 & 0.0328 & -0.1811 & No & No \\
\hline Reference height & 0.0058 & 0.2732 & 0.5243 & No & No \\
\hline FSM discriminant function & 0.0823 & 3.8686 & 2.0532 & $\mathrm{P}<0 . \mathrm{I}$ & $\mathrm{P}<0.05$ \\
\hline RB discriminant function & 0.0517 & 2.4277 & 1.6000 & No & No \\
\hline Cup area temporal & 0.0582 & 2.7360 & -1.7044 & $P \approx 0.1$ & $\mathrm{P}<0.1$ \\
\hline
\end{tabular}

(Continued) 
Table 2 (Continued)

\begin{tabular}{|c|c|c|c|c|c|}
\hline Parameter & $R^{2}$ & F-test & t-test & F-test (satisfied) & t-test (satisfied) \\
\hline Cup area temp/sup & 0.0574 & 2.6984 & -1.6920 & $\mathrm{P} \approx 0.1$ & $\mathrm{p}<0.1$ \\
\hline Cup area temp/inf & 0.1103 & 5.1825 & -2.4134 & $\mathrm{p}<0.05$ & $\mathrm{P}<0.05$ \\
\hline Cup area nasal & 0.1213 & 5.6991 & -2.5467 & $\mathrm{p}<0.05$ & $\mathrm{p}<0.05$ \\
\hline Cup area nasal/sup & 0.0649 & 3.0505 & -1.8062 & $\mathrm{P}<0 . \mathrm{I}$ & $\mathrm{p}<0.1$ \\
\hline Cup area nasal/inf & 0.1352 & 6.3532 & -2.7104 & $\mathrm{P}<0.05$ & $\mathrm{p}<0.01$ \\
\hline Cup volume temporal & 0.0868 & 4.0803 & -2.1138 & $\mathrm{P} \approx 0.05$ & $\mathrm{P}<0.05$ \\
\hline Cup volume temp/sup & 0.0659 & 3.0992 & -1.8215 & $\mathrm{P}<0.1$ & $\mathrm{p}<0.1$ \\
\hline Cup volume temp/inf & 0.1386 & 6.5143 & -2.7500 & $\mathrm{p}<0.05$ & $\mathrm{p}<0.01$ \\
\hline Cup volume nasal & 0.0804 & 3.7769 & -2.0266 & $\mathrm{p}<0.1$ & $\mathrm{P} \approx 0.05$ \\
\hline Cup volume nasal/sup & 0.0426 & 2.0007 & -1.4456 & No & No \\
\hline Cup volume nasal/inf & 0.1205 & 5.6637 & -2.5377 & $\mathrm{P}<0.05$ & $\mathrm{P}<0.05$ \\
\hline CSM temporal & 0.0446 & 2.0958 & -1.48|| & No & No \\
\hline CSM temp/sup & 0.0085 & 0.3987 & $-0.634 I$ & No & No \\
\hline CSM temp/inf & 0.0756 & 3.5512 & -1.9600 & $\mathrm{p}<0.1$ & $\mathrm{p}<0.1$ \\
\hline CSM nasal & 0.0021 & 0.0978 & -0.3130 & No & No \\
\hline CSM nasal/sup & 0.0176 & 0.8256 & -0.9167 & No & No \\
\hline CSM nasal/inf & 0.0066 & 0.3082 & -0.5570 & No & No \\
\hline Diagnosis & 0.1113 & 5.2317 & -2.4263 & $\mathrm{P}<0.05$ & $\mathrm{P}<0.05$ \\
\hline MRA & 0.1100 & 5.1702 & -2.4102 & $\mathrm{p}<0.05$ & $\mathrm{P}<0.05$ \\
\hline
\end{tabular}

Notes: *The last two variables (diagnosis, MRA) are linguistic ones, so it was necessary to modify them. ${ }^{\dagger}$ The values of diagnosis can be PXF, POAG, PG, NTG, which are represented by the arithmetic values I to 4 , respectively. This variable is omitted in the cases of different models for each kind of glaucoma diagnosis. ${ }^{\ddagger}$ The MRA values can be within normal limits, borderline, outside normal limits, which are represented by the arithmetic values I to 3 , respectively.

Abbreviations: CCT, central corneal thickness; CSM, cup shape measure; CLM, contour line modulation; IOP, intraocular pressure; FSM, Frederick S Mikelberg discriminant function; MD, mean defect; MRA, Moorfields regression analysis; NTG, normal tension glaucoma; RB, Renuka Bathija; RNFL, retinal nerve fiber layer; PG, pigmentary glaucoma; POAG, primary open-angle glaucoma; PXF, pseudoexfoliative glaucoma.

relationship between CCT and IOP may have been affected by the fact that at the time of initial diagnosis, glaucomatous eyes with high pretreatment IOPs, due to high CCT, might have received more aggressive treatment, resulting in lower IOPs despite the presence of thick cornea and vice versa. As a result, there is no dependence between CCT and IOP measurements in our selected group of patients.

If we compare the results of simple linear monovariable regression model between each input variable and CCT using the $\mathrm{R}^{2}$ criterion (which is registered in Table 2) with the respective results of the proposed model, the new method provides significantly higher $\mathrm{R}^{2}$ values for our selected group of patients. $\mathrm{R}^{2}$ has been improved by $46 \%$ against the best simple regression model with input parameter cup area nasal, which has $\mathrm{R}^{2}$ equal to 0.1213 . This optimal result is not achieved by just adding new variables during the model fitting analyses. The best model is justified as the one with the highest $\mathrm{R}^{2}$ that at the same time satisfies both the following statements:

- Provides statistically significant $p_{1}$ values $\left(p_{1}<0.05\right)$ for the model F-test.
- Provides statistically significant $p_{2}$ values $\left(p_{2}<0.05\right)$ for the t-test of each one of the included variables.

Our study was limited by the fact that the sample size was relatively small. The sample size and the characteristics of subjects have an influence on the results. Thus, although the results of the present study provide information on associations in our selected group of patients, a possible selection bias may account for these associations and a larger population-based study should be performed to confirm these findings.

In conclusion, this paper presents a nonlinear multivariable regression method, with which the model of equation (1) has been constructed, describing the relationship between CCT and $\mathrm{ONH}$ structural parameters in eyes with established glaucoma. Our findings suggest that this relationship is far more complex than can be modeled by simple linear arithmetic formulas. By using the new nonlinear multivariate model in eyes with established glaucoma, our study showed that CCT was significantly associated with the HRTII structural measurement rim volume nasally. The fact that no correlation was found between CCT and IOP possibly 


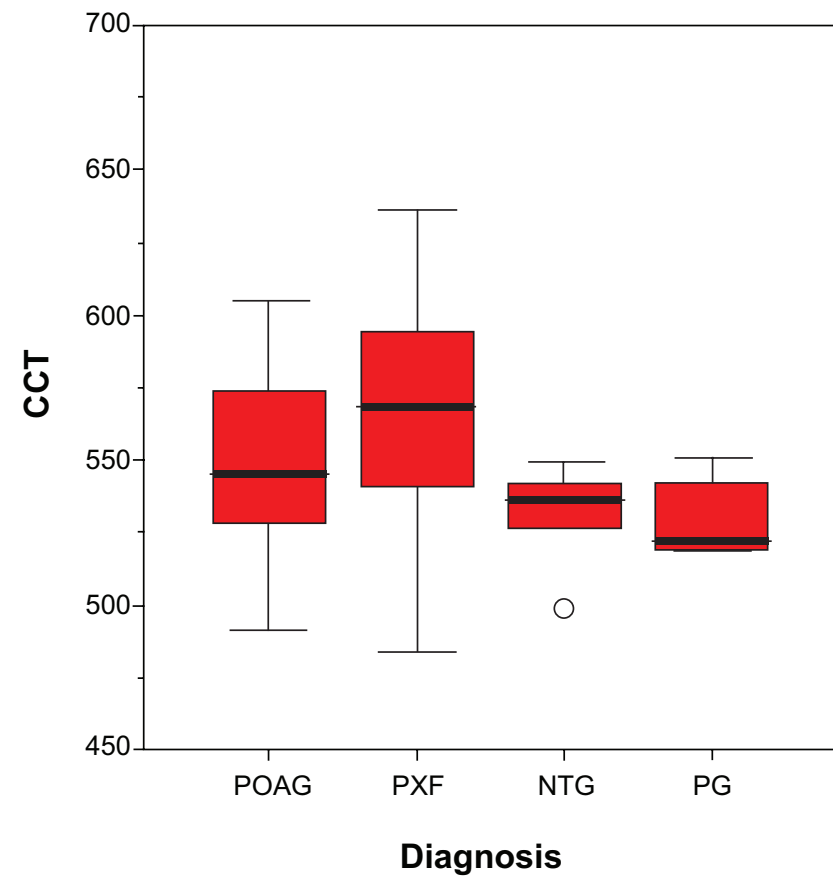

Figure 3 Distribution of corneal thickness according to diagnosis (POAG, NTG, PXF, PG).

Abbreviations: CCT, central corneal thickness; NTG, normal tension glaucoma; PG, pigmentary glaucoma; POAG, primary open-angle glaucoma; PXF, pseudoexfoliative glaucoma.

reflects the effect of differing treatment regimes on patients' eyes. Longitudinal data collection should be performed to confirm these findings.

\section{Acknowledgments}

Presented in part at the 3rd International Congress on Glaucoma Surgery, Toronto, ON, Canada, May 25-28, 2006, and the 1st WSEAS International Conference on Biomedical Electronics and Biomedical Informatics (BEBI '08) Rhodes, Greece, August 20-22, 2008.

\section{Disclosure}

The authors report no conflicts of interest in this work.

\section{References}

1. Gordon MO, Beiser JA, Brandt JD, et al. The Ocular Hypertension Treatment Study: baseline factors that predict the onset of primary open-angle glaucoma. Arch Ophthalmol. 2002;120:714-720.

2. Medeiros FA, Sample PA, Weinreb RN. Corneal thickness measurements and frequency doubling technology perimetry abnormalities in ocular hypertensive eyes. Ophthalmology. 2003;110:1903-1908.

3. Medeiros FA, Sample PA, Zangwill LM, Bowd C, Aihara M, Weinreb RN. Corneal thickness as a risk factor for visual field loss in patients with preperimetric glaucomatous optic neuropathy. $\mathrm{Am} \mathrm{J}$ Ophthalmol. 2003;136:805-813.

4. Kim JW, Chen PP. Central corneal pachymetry and visual field progression in patients with open-angle glaucoma. Ophthalmology. 2004;111:2126-2132.
5. Quigley HA, Addicks EM, Green WR. Optic nerve damage in human glaucoma. III. Quantitative correlation of nerve fiber loss and visual field defect in glaucoma, ischemic neuropathy, papilledema and toxic neuropathy. Arch Ophthalmol. 1982;100:135-146.

6. Tuulonen A, Airaksinen PJ. Initial glaucomatous optic disc and retinal fiber layer abnormalities and their progression. Am J Ophthalmol. 1991;111:485-490.

7. Sommer A, Katz J, Quigley HA, et al. Clinically detectable nerve fiber atrophy precedes the onset of glaucomatous field loss. Arch Ophthalmol. 1991;109:77-83.

8. Johnson CA, Sample PA, Cioffi GA, et al. Structure and function evaluation (SAFE): I. criteria for glaucomatous visual field loss using standard 14 automated perimetry (SAP) and short wavelength automated perimetry (SWAP). Am J Ophthalmol. 2002;134(2):177-185.

9. Johnson CA, Sample PA, Zangwill LM, et al. Structure and function evaluation (SAFE): II. Comparison of optic disk and visual field characteristics. Am J Ophthalmol. 2003;135(2):148-154.

10. Ugurlu S, Hoffman D, Garway-Heath DF, et al. Relationship between structural abnormalities and short-wavelength perimetric defects in eyes at risk of glaucoma. Am J Ophthalmol. 2000;129:592-598.

11. Herndon LW, Weizer JS, Stinnett SS. Central corneal thickness as a risk factor for advanced glaucoma damage. Arch Ophthalmol. 2004;122:17-21.

12. Hewitt AW, Cooper RL. Relationship between corneal thickness and optic disc damage in glaucoma. Clin Experiment Ophthalmol. 2005;33:158-163.

13. Hasslett RS, Batterbury M, Cuypers M, Cooper RL. Interobserver agreement in clinical optic disc measurement using a modified $60 \mathrm{D}$ lens. Eye. 1997;11:692-697.

14. Jonas JB, Stroux A, Velten I, Juenemann A, Martus P, Budde WM. Central corneal thickness correlated with glaucoma damage and rate of progression. Invest Ophthalmol Vis Sci. 2005;46(4):1269-1274.

15. Sihota R, Gulati V, Agarwal HC, et al. Variables affecting test-retest variability of Heidelberg Retina Tomograph II stereometric parameters. J Glaucoma. 2002;11:321-328.

16. Verdonck N, Zeyen T, Van Malderen L, et al. Short-term intraindividual variability in Heidelberg Retina Tomograph II. Bull Soc Belge Ophtalmol. 2002;(286):51-57.

17. Strouthidis NG, White ET, Owen VMF, Ho TA, Hammond CJ, and Garway-Heath DF. Factors affecting the test-retest variability of Heidelberg retina tomograph and Heidelberg retina tomograph II measurements. Br J Ophthalmol. 2005;89:1427-1432.

18. Sigal IA, Flanagan JG, Ethier CR. Factors influencing optic nerve head biomechanics. Invest Ophthalmol Vis Sci. 2005;46:4189-4199.

19. Sigal IA, Flanagan JG, Tertinegg I, Ethier CR. Finite element modeling of optic nerve head biomechanics. Invest Ophthalmol Vis Sci. 2004;45:4378-4387.

20. Sigal IA, Flanagan JG, Tertinegg I, Ethier CR. Reconstruction of human optic nerve heads for finite element modeling. Technol Health Care. 2005; 13:313-329.

21. Hand D, Manilla H, Smyth P. Principles of Data Mining. Cambridge, MA: The MIT Press; 2001. p. 368-371.

22. Tsekouras GJ, Elias CN, Kavatza S, Contaxis GC. A hybrid non-linear regression midterm energy forecasting method using data mining. 2003 IEEE Bologna Power Tech Conference, June 23-26th, Bologna, Italy.

23. Tsekouras GJ, Dialynas EN, Hatziargyriou ND, Kavatza S. A non-linear multivariable regression model for midterm energy forecasting of power systems. Electr Power Syst Res. 2007;77(12):1560-1568.

24. European Glaucoma Society. Terminology and Guidelines for Glaucoma. Savona: Editrice Dogma; 2003. p. 24-32.

25. Jonas JB, Fernandez MC, Stürmer J. Pattern of glaucomatous neuroretinal rim loss. Ophthalmology. 1993;100(1):63-68.

26. Pederson JE, Anderson DR. The mode of progressive disc cupping in ocular hypertension and glaucoma. Arch Ophthalmol. 1980;98:490-495.

27. Caprioli J, Miller JM, Sears M. Quantitative evaluation of the optic nerve head in patients with unilateral visual field loss from primary open-angle glaucoma. Ophthalmology. 1987;94:1484-1487. 
28. Garway-Heath DF, Hitchings RA. Quantitative evaluation of the optic nerve head in early glaucoma. Br J Ophthalmol. 1998;82:352-361.

29. Copt RP, Thomas R, Mermoud A. Corneal thickness in ocular hypertension, primary open angle glaucoma and normal tension glaucoma. Arch Ophthalmol. 1999;117:14-16.

30. Emara BY, Tingey DP, Probst LE, Motolko MA. Central corneal thickness in low-tension glaucoma. Can J Ophthalmol. 1999;319-324.

31. Pakravan M, Parsa A, Sanagou M, Parsa CF. Central corneal thickness and correlation to optic disc size: a potential link for susceptibility to glaucoma. Br J Ophthalmol. 2007;91(1):26-28.

32. Uchida H, Brigatti L, Caprioli J, et al. Detection of structural damage from glaucoma with confocal laser image analysis. Invest Ophthalmol Vis Sci. 1996;37:2393-2401.

33. Wollstein G, Garway-Heath DF, Hitchings RA, et al. Identification of early glaucoma cases with the scanning laser ophthalmoscope. Ophthalmology. 1998;105:1557-1563.

34. Iester M, Mikelberg FS, Swindale NV, Drance SM. ROC curve analysis of Heidelberg Retina Tomograph optic disc shape measures in glaucoma. Can J Ophthalmol. 1997;32:382-388.

35. Argus WA. Ocular hypertension and central corneal thickness. Ophthalmology. 1995;102:1810-1812.
36. Bron AM, Creuzot-Garcher C, Goudeau-Boutillon S, d'Athis P. Falsely elevated intraocular pressure due to increased central corneal thickness. Graefes Arch Clin Exp Ophthalmol. 1999;237:220-224.

37. Copt RP, Thomas R, Mermoud A. Corneal thickness in ocular hypertension, primary open-angle glaucoma, and normal tension glaucoma. Arch Ophthalmol. 1999;117:14-16.

38. Herndon LW, Choudhri SA, Cox T, Damji KF, Shields MB, Allingham RR. Central corneal thickness in normal, glaucomatous, and ocular hypertensive eyes. Arch Ophthalmol. 1997;115:1137-1141.

39. Herman DC, Hodge DO, Bourne WM. Increased corneal thickness in 17 patients with ocular hypertension. Arch Ophthalmol. 2001;119: 334-336.

40. Whitacre MM, Stein R. Sources of error with use of Goldmanntype tonometers. Surv Ophthalmol. 1993;38:1-30.

41. Whitacre MM, Stein RA, Hassanein K. The effect of corneal thickness on applanation tonometry. Am J Ophthlmol. 1993;115:592-596.

42. Lleo A, Marcos A, Calatayud M, Alonso L, Rahhal SM, SanchisGimeno JA. The relationship between central corneal thickness and Goldmann applanation tonometry. Clin Exp Optom. 2003;86:104-108. 


\section{Appendix}

The method assumes that a particular set of variables has been selected through doctor's knowledge, data preprocessing, and conversion. These variables will be examined for potential incorporation in the model. The selected set of variables can be structured in vectors as:

$$
\vec{x}_{i}=\left(x_{i 1}, \quad x_{i 2}, \quad \ldots, \quad x_{i n}\right)^{T}=\left(x_{i j}, j=1, \ldots, n\right)^{T}
$$

where $x_{i j}$ is the value of the $j$-th selected variable for the eye $i$. There are $m_{1}$ vectors for training the model and $m_{2}$ for conducting the final estimation of CCT.

Figure 1 shows the basic steps of the developed method, which has the following main components:

- proper transformation of the model variables,

- use of correlation analysis,

- model optimization regarding the selection of input variables and the application of the validation criterions.

The nonlinear functions to be used by the method belong to a selected function set $F=\left\{f_{k}\left(x_{j}\right): k=1 \ldots K\right\}$, where $x_{j}$ is the $j$-th selected variable. Such functions can be $x^{a}, 1 / x$, $\ln (x), e^{-x}$, while the parameter $a$ belongs to specific set A and should be determined. Based on the selected function set $F$ the basic vector $\vec{X}_{i}$ is defined as:

$$
\vec{X}_{i}=\left(1 \quad f_{1}\left(x_{i 1}\right) \ldots f_{K}\left(x_{i 1}\right), \ldots f_{1}\left(x_{i N}\right) \ldots f_{K}\left(x_{i N}\right)\right)^{T}
$$

having dimension $w$ equal to $1+N \cdot K$, where $N$ is the number of the input variables, which are pre-selected by the user's knowledge or by simple data mining techniques such as two dimensional scatter-plot analysis etc. Any linear combination of terms contained in vector $\vec{X}_{i}$ forms a basis for a candidate estimation model. The number of all possible combinations is $2^{N \cdot K}$, since the constant term always exists.

In order to reduce the number of candidate combinations, a correlation analysis is performed using the following basic steps:

- The correlation index between $f_{k}\left(x_{j}\right)$ and $y$ is computed and the term $f_{k}\left(x_{j}\right)$ is retained for further processing, if the index is greater than a pre-specified value cor $_{1}$.

- For all terms being retained, a cross correlation analysis is performed. If the correlation index between any two terms $f_{k}\left(x_{j}\right)-f_{k^{\prime}}\left(x_{j^{\prime}}\right)$, for $k \neq k^{\prime}$ and $j \neq j^{\prime}$ simultaneously, is smaller than a pre-specified value cor $_{2}$, both terms are retained, otherwise only the term with the largest correlation with respect to output $y$ is retained. Therefore, all functions that have information overlap are deduced through this cross correlation analysis and form the set $F_{c}$.
- The function having the smaller absolute correlation index with the variable $y$ is removed and this process is repeated until the set $F_{c}$ is empty.

The above analysis results in a reduced subset of $w_{1}$ variables $X_{i j}$, which form the vector $\vec{X}_{t-t}$. Any linear combination of any of the $w_{1}$ variables included in the vector $\vec{X}_{t-t}\left(2^{w_{1}}\right.$ such combinations) is a candidate estimation model. All these combinations should be examined in order to determine the one leading to the best estimation.

If $\vec{X}_{t}$ is one of the combinations having dimension $w_{2}$, the nonlinear multivariable regression model has the following form:

$$
\tilde{y}_{t}=\vec{b}^{T} \cdot \vec{X}_{t}
$$

where $\vec{b}=\left(b_{1}, b_{2}, \ldots, b_{w_{2}}\right)^{T}$ is the unknown vector of constant coefficients, $\tilde{y}_{t}$ is the output variable to be estimated and $y_{t}$ is the respective real one. The estimated vector $\tilde{\vec{b}}$ and the respective covariance matrix $\operatorname{cov}(\tilde{\vec{b}})$ are given by:

$$
\begin{gathered}
\tilde{\vec{b}}=\left(X_{P}^{T} \cdot X_{P}\right)^{-1} \cdot X_{P}^{T} \cdot \vec{Y} \\
\operatorname{cov}(\tilde{\vec{b}})=\sigma_{v}^{2} \cdot\left(X_{P}^{T} \cdot X_{P}\right)^{-1}
\end{gathered}
$$

where $\sigma_{v}^{2}=\sum_{t=1}^{m_{1}} e_{t}^{2} /\left(m_{1}-w_{2}\right), \vec{Y}=\left(y_{1}, y_{2}, \ldots, y_{m_{1}}\right)^{T}$ is the vector of the real output values, $X_{P}=\left(\vec{X}_{1}, \vec{X}_{2}, \ldots, \vec{X}_{m_{1}}\right)^{T}$ is the matrix of the input variables. The procedure for the mathematical solution of this problem is presented by Tsekouras and colleagues..$^{20}$

If the vector $\vec{X}_{t}^{*}$ of the selected variables is known for the eye $t$, the mean value and the covariance of the central corneal thickness are given by:

$$
\begin{gathered}
y_{t}^{*}=\tilde{\vec{b}}^{T} \cdot \vec{X}_{t}^{*} \\
\sigma_{y_{t}^{*}}^{2}=\vec{X}_{t}^{* T} \cdot \operatorname{cov}(\tilde{\vec{b}}) \cdot \vec{X}_{t}^{*}
\end{gathered}
$$

The validation criterions are the $F$-test and the coefficient determination $R^{2}$ for the regression model and $t$-tests for the $b_{j}$ parameters, which are given by:

$$
\begin{gathered}
F=\frac{\sum_{i=1}^{m_{1}}\left(\bar{y}-\tilde{y}_{i}\right)^{2} /\left(w_{2}-1\right)}{\sum_{i=1}^{m_{1}}\left(y_{i}-\tilde{y}_{i}\right)^{2} /\left(m_{1}-w_{2}\right)} \\
R^{2}=\frac{\sum_{i=1}^{m_{1}}\left(\bar{y}-\tilde{y}_{i}\right)^{2}}{\sum_{i=1}^{m_{1}}\left(\bar{y}_{i}-y_{i}\right)^{2}}
\end{gathered}
$$




$$
t_{\left(m_{2}-w_{2}\right)}-j=\frac{b_{j}}{\sqrt{\operatorname{cov}(\tilde{\vec{b}})_{j, j}}}
$$

Therefore, the main steps of the developed regression method are the following:

1. The $N$ input variables are selected using simple data mining techniques, such as scatter-plot analysis between each possible input variable and the forecasting one. Alternatively the user's knowledge can be used.

2. Based on the selected variables and function $\operatorname{set} F$, the basic vector $\vec{X}_{i}$ is determined.

3. Based on correlation analysis, a reduced subset of terms of the basic vector $\vec{X}_{i}$ is selected to form the vector $\vec{X}_{t-t}$.

4. Any combination of the terms in $\vec{X}_{t-t}$ is a vector $\vec{X}_{i}$, candidate to be examined as a basis for the estimation model.
5. For each vector $\vec{X}_{i}$ the three validation criterions are calculated ( $F$-test, $R^{2}, t$-test).

6. The $F$-test will be successful, if the $F$-value by eq. (8) is greater than the respective value of the Snedecor probability distribution for given probability $p_{1}$, with $v_{2}=m_{1}-1, v_{1}=w_{2}-1$. The $t$-test will be successful, if the $t$-value for each $b_{j}$ parameter by eq. (10) is greater than (or at least equal to) the respective value of the Student probability distribution for given probability $p_{2}$, with $v_{1}=m_{1}-w_{2}$. The $\vec{X}_{i}$ is a successful vector, if both of the $F$-test and $t$-test for all $b_{j}$ are successful.

7. The vector $\vec{X}_{i}$ with the overall minimum value of $R^{2}$ is selected. 
